\title{
The assessment of glomerular function: creatinine clearance or plasma creatinine?
}

\author{
D. B. MORGAN \\ M.D., M.R.C.Path.
}

\author{
S. Dillon \\ B.Sc., M.B., B.Ch.
}

\author{
R. B. PAYNe \\ M.D., Ph.D., F.R.C.Path. \\ Departments of Chemical Pathology, General Infirmary, Leeds, and \\ St James's Hospital, Leeds
}

\begin{abstract}
Summary
Plasma creatinine concentration is superior to creatinine clearance for the detection of abnormal glomerular function, of changes of glomerular function in patients with chronic renal disease. Creatinine clearance should be abandoned as the routine assessment of glomerular function.

\section{Introduction}

In clinical practice, the assessment of glomerular function or overall renal function in patients thought or known to have chronic renal disease is based on some measure of glomerular filtration rate (GFR).

It is generally accepted that gross reduction in glomerular function can be detected by measuring plasma creatinine alone. However, Tobias, McLaughlin and Hopper (1962) and Doolan, Alpen and Theil (1962) concluded that in the detection of smaller reductions of glomerular function, creatinine clearance had worthwhile advantages over the much simpler measurement of plasma creatinine, and this view has generally persisted. The authors have reconsidered the arguments and assessed the quality of the information obtained about glomerular function with the two measures, creatinine clearance and plasma creatinine. Their major conclusion is that the routine measurement of endogenous creatinine clearance should be abandoned and efforts should be concentrated on improving the information which can be obtained from the measurement of plasma creatinine.
\end{abstract}

What clinicians want to know about glomerular function

It is not very often that a clinician wants to know a patient's GFR. Perhaps the only situation is when the required dose of a drug depends on glomerular function.

Correspondence: Dr D. B. Morgan, Department of Chemical Pathology, Martin Wing, General Infirmary, Leeds.
The more usual questions are whether glomerular function is abnormal, to what extent it is abnormal, and whether it has changed. In practice a patient's value in health is usually not known so that the presence of abnormality and its extent have to be assessed by comparing his value for the chosen test with the appropriate reference range. The reliability of the decisions about normality or change of glomerular function also depends on the reproducibility of the measurement, that is, the variability in the test result in the absence of a real change. Thus the problems of reference ranges and of reproducibility are important aspects of the assessment o glomerular function.

\section{The tests of glomerular function}

Four tests are used to assess glomerular function; plasma urea, plasma creatinine, endogenous creatinine clearance and the clearance of an injected compound such as inulin or ${ }^{51} \mathrm{Cr}$-EDTA. Inulin and ${ }^{51}$ Cr-EDTA clearance give a more accurate assessment of GFR than does creatinine clearance because of the tubular secretion of creatinine (Materson, 1971). However, these techniques are more complex and less freely available than creatinine clearance and, although they give more accurate and precise information, the problems of interpretation are the same as with creatinine clearance.

At the other extreme, the simple and readily available measurement of plasma urea is influenced by several factors other than changes in glomerular function, such as changes in protein intake and in urine flow rate, which do not significantly affect plasma creatinine (Kassirer, 1971; Kerr and Davidson, 1975). A study by the authors has shown a very wide range of relations between plasma urea and plasma creatinine concentrations in a sequential series of hospital patients.

Plasma creatinine is becoming increasingly available on a same day or even an emergency basis and there can be little doubt that it should replace urea as the screening procedure for renal failure. The 
question which remains is whether, in routine clinical practice, creatinine clearance has clear-cut advantages over plasma creatinine for the detection of abnormal glomerular function and of changes in glomerular function. The choice will depend not only on the physiological relation between plasma creatinine and creatinine clearance but also on the quality of the available reference ranges and the reproducibility of the measurements.

\section{The relation between plasma creatinine and creatinine clearance}

Plasma creatinine concentration (P) is determined by the creatinine production rate and by the creatinine clearance (Ccr). In the steady state, creatinine production rate and excretion rate are equal (Payne and Morgan, 1977) and can be measured as the product of urine creatinine concentration and urine volume per unit time (U.V). But Ccr equals U.V/P so that P equals U.V/Ccr. Plasma creatinine (P) therefore increases as creatinine production (U.V) increases or creatinine clearance (Ccr) decreases. It follows that in an individual with a constant rate of creatinine production (U.V), P will vary as the reciprocal of Ccr. Any reduction of creatinine clearance in an individual is associated with an increase in $P$, and the rate of increase will accelerate as GFR falls progressively; a halving of Ccr will cause a doubling of $\mathbf{P}$. Figure 1 shows the relation between $\mathbf{P}$ and $\mathrm{Ccr}$ as

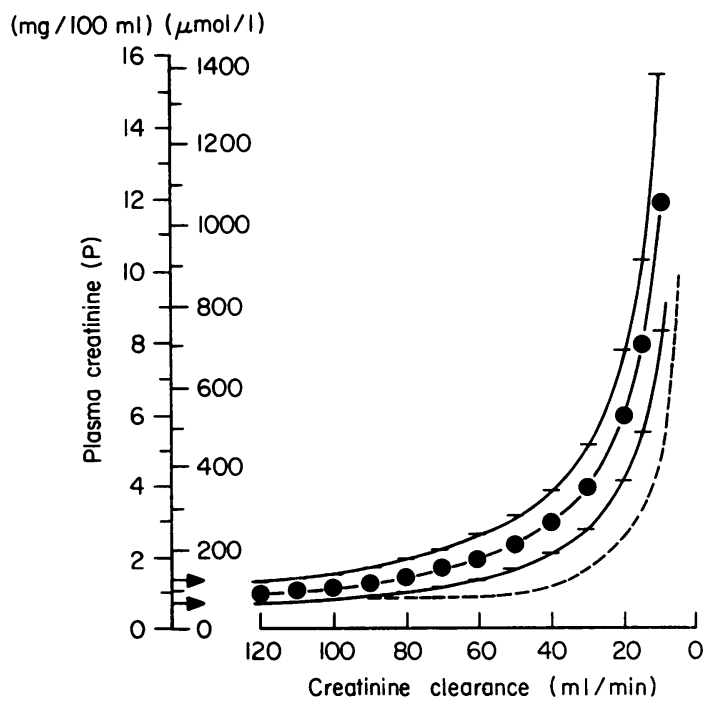

FIG. 1. The relation between plasma creatinine and creatinine clearance $(\mathrm{ml} / \mathrm{min})$. The continuous lines show the calculated relationship in young men as creatinine clearance falls (assuming $U . V$ is constant). The interrupted lines indicate the approximate extreme relationship which might be observed in healthy elderly men.
Ccr decreases in a group of young persons and in elderly persons.

\section{Technique and reference ranges}

There is a large literature on the specificity of the various analytical techniques used to measure plasma and urine creatinine. Nearly all clinical chemistry laboratories use methods based on the Jaffé reaction (Cook, 1975). This reaction is not specific for creatinine because of non-creatinine chromogens in plasma. The technique can be made more specific by using an absorbent such as Fuller's earth but in practice most laboratories use the Jaffé reaction on an AutoAnalyser which gives reproducible results close to those obtained with Fuller's earth.

Plasma creatinine has been measured in large groups of apparently well persons, but few studies have examined the changes with age in detail. Figure 2 shows that the average value for $P$ increases through childhood to reach adult values by about the age of 20 years (Kuhlback, Eriksson and Forsius, 1964; Donckerwolcke et al., 1970; Schwartz et al., 1976).

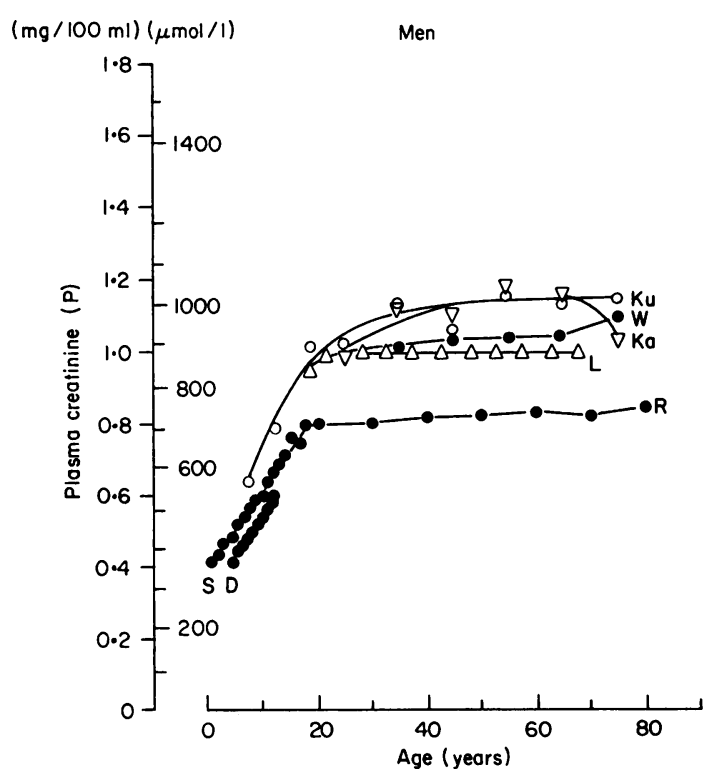

FIG. 2. The plasma creatinine in healthy men according to age. The data are taken from: (D) Donckerwolcke et al. 1970; (Ka) Kampmann et al. 1971; (Ku) Kuhlback et al. 1964; (L) de Lauture et al. 1973; (R) Rowe et al. 1976; (S) Schwartz et al. 1976; (W) Wilding et al. 1972.

In studies of large numbers of apparently healthy adults the $P$ increases by only a few per cent between 20 and 80 years (Kuhlback et al., 1964; Wilding, Rollason and Robinson, 1972; de Lauture et al., 1973). The range of values is about the same at all 
ages. Rowe et al. (1976) studied men in the Baltimore Longitudinal Study of Aging and excluded patients with evidence of disease (336 of 884); many of these excluded patients had evidence of renal disease. Figure 2 shows that the $P$ did not change with age in the remaining 'normal' men. Kampmann et al. (1972) studied unselected patients in the medical wards of three hospitals. They excluded patients with a $P \geqslant 135$ $\mu \mathrm{mol} / \mathrm{l}$ and the $\mathbf{P}$ in the remaining patients did not change with age (Fig. 2). In these two studies (Kampmann et al., 1971; Rowe et al., 1976), the frequency of exclusion increased markedly with age indicating that the prevalence of renal disease increased with age. Cockroft and Gault (1976) studied the plasma creatinine in hospital patients who had had their creatinine clearance measured for diagnostic reasons. The $\mathbf{P}$ in these patients increased with age presumably because of the inclusion of patients with renal disease the prevalence or which increases with age, if one takes into account hypertensive vascular disease.

The results taken together suggest that in the healthy population there is little increase in the average $P$ with age. However, the frequency of renal disease increases with age so that, in unselected patients in hospitals, the average $P$ increases with age.

The relation between $P$ and age is similar in women to that in men but the values in adult women are about $10 \%$ lower than those in men at all ages (Table 1). This is about the same as the percentage difference in lean body mass between men and women, so that the average ratio of $P$ to lean body mass is about the same in men and women (Doolan et al., 1962). However, it has not been shown that there is a relation between $P$ and lean body mass within a group of men and women.

There have been three studies of the relation between Ccr and age (Cockroft and Gault, 1976; Kampmann et al., 1971; Rowe et al., 1976). Figure 3 shows that in these studies Ccr in men falls by as much as $50 \%$ between the ages of 20 and 70 years. In two of these studies, men with renal disease were excluded (Kampmann et al., 1971; Rowe et al., 1976). In the third study (Cockroft and Gault, 1976), the men were being investigated for renal disease, and the Ccr was lower than in the other two studies, particularly in the older men where uro-genital disease is most common. The percentage fall with age is about the same in women as in men (Kampmann et al., 1971). The results taken together suggest that there is a progressive reduction in Ccr (glomerular function) with age even in the patients without clearcut evidence of renal disease whose $P$ does not increase with age (Fig. 3). The constancy of $P$ in the face of a fall in Ccr is explained by a fall in creatinine production (U.V) which is proportionally similar to the fall in Ccr (Fig. 4).

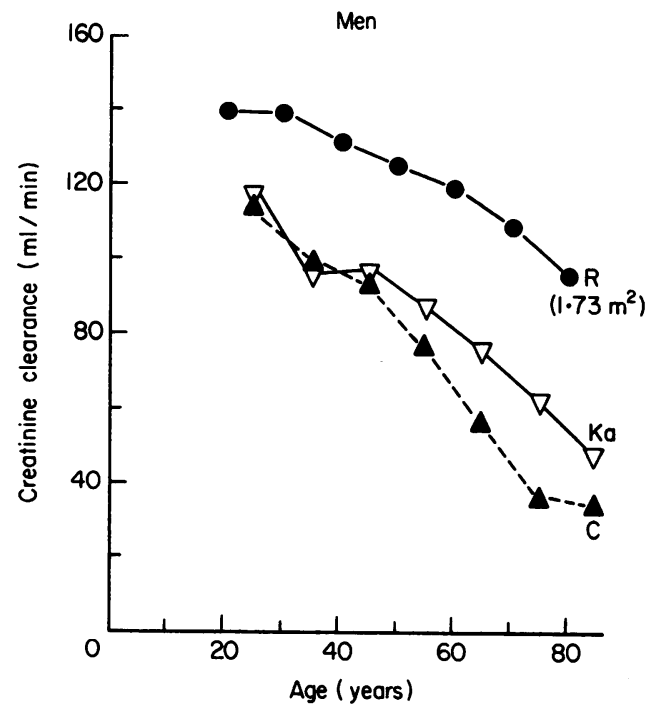

FIG. 3. The creatinine clearance $(\mathrm{ml} / \mathrm{min})$ in healthy men according to age. The data are taken from: (C) Cockroft and Gault 1976; (Ka) Kampmann et al. 1971 and (R) Rowe et al. 1976.

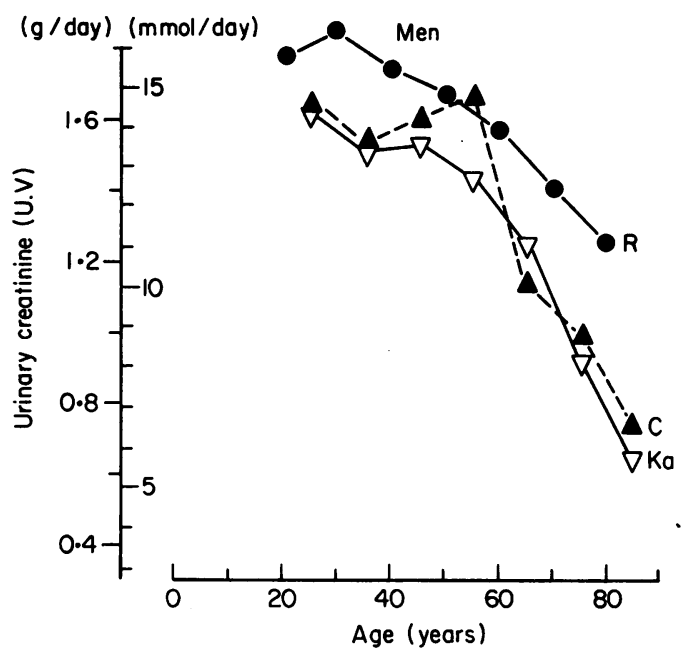

FIG. 4. The daily urinary excretion of creatinine in healthy men according to age. The data are taken from: (C) Cockroft and Gault 1976; (Ka) Kampmann et al. 1971 and (R) Rowe et al. 1976.

It is generally assumed that there is a relation between creatinine clearance and body size, but this relationship has not been defined in detail in adults. The standardization of $\mathrm{Ccr}$ in terms of calculated body surface area was originally suggested as a means of giving similar 'normalized' values in children and adults (McIntosh, Moller and van Slyke, 1928). There was no significant relation 
TABLE 1. Some reference ranges for plasma creatinine ( $\mu \mathrm{mol} / \mathrm{l})$

\begin{tabular}{|c|c|c|c|c|c|c|c|c|}
\hline \multirow{3}{*}{\multicolumn{2}{|c|}{ Reference }} & \multirow[b]{3}{*}{ Method* } & \multicolumn{3}{|c|}{ Men } & \multicolumn{3}{|c|}{ Women } \\
\hline & & & \multirow{2}{*}{$\begin{array}{c}20-30 \text { years } \\
\bar{x} \text { (s.d.) }\end{array}$} & \multicolumn{2}{|c|}{ All ages } & \multirow{2}{*}{$\begin{array}{c}20-30 \text { years } \\
\bar{x} \text { (s.d.) }\end{array}$} & \multicolumn{2}{|c|}{ All ages } \\
\hline & & & & Ages & $\bar{x}$ (s.d.) & & Ages & $\bar{x}$ (s.d.) \\
\hline 1 & Doolan et al. (1952) & True & & $21-59$ & $89(10)$ & & $21-59$ & $72(8)$ \\
\hline 2 & Tobias et al. (1962) & Tot. & & $19-52$ & 112 (11) & & $19-52$ & $96(12)$ \\
\hline 3 & Enger and Blegen (1964) & Tot. & & $19-63$ & 99 (10) & & $20-58$ & 89 (11) \\
\hline 4 & Kuhlback et al. (1964) & Tot. & $88(5)$ & $5-70$ & $92(2)$ & $82(3)$ & $5-70$ & 83 (1) \\
\hline 5 & Kampmann et al. (1971) & Tot. & 88 (14) & & & 79 (15) & & \\
\hline 6 & Wilding et al. (1972) & Tot. & $89(14)$ & & & $70(15)$ & & \\
\hline 7 & de Lauture et al. (1973) & True & $88(11)$ & $15-59$ & $88(12)$ & 78 (11) & $15-59$ & 79 (11) \\
\hline 8 & Cockroft and Gault (1976) & AA & 88 & & & & & \\
\hline 9 & Rowe et al. (1976) & True & $71(8)$ & & & & & \\
\hline
\end{tabular}

*Method: True, creatinine measured after absorption with Fuller's Earth. Tot., total chromogens. AA, Autoanalyser method.

TABLE 2. Some reference ranges for creatinine clearance in young adults $(\mathrm{ml} / \mathrm{min})$

\begin{tabular}{|c|c|c|c|c|}
\hline Reference & Method* & Ages (years) & $\begin{array}{c}\text { Men } \\
\bar{x} \text { (s.d.) }\end{array}$ & $\begin{array}{l}\text { Women } \\
\bar{x} \text { (s.d.) }\end{array}$ \\
\hline $\begin{array}{ll}1 & \text { †Doolan et al. }(1962) \\
2 & \text { Tobias et al. }(1962) \\
3 & \text { Kampmann et al. }(1971) \\
4 & \text { Cockroft and Gault (1976) } \\
5 & \text { Rowe } \text { et al. }(1976)\end{array}$ & $\begin{array}{l}\text { Tot. } \\
\text { Tot. } \\
\text { Tot. } \\
\text { AA } \\
\text { True }\end{array}$ & $\begin{array}{r}17-50 \\
19-52 \\
20-29 \\
18-29 \\
25-34\end{array}$ & $\begin{array}{l}120(25 \cdot 5) \\
105(14) \\
117(23) \\
115- \\
140(21) \ddagger\end{array}$ & $\begin{array}{l}96(13 \cdot 5) \\
95(18) \\
91(19) \\
--\end{array}$ \\
\hline
\end{tabular}

* As Table 1.

$\dagger$ Based on mean of three measurements in each person.

$\ddagger$ 'Corrected' to surface area $1.73 \mathrm{~m}^{2}$.

between calculated surface area and Ccr in adults. Tables 1 and 2 show the reported reference ranges for $P$ and $C c r$ in young men and women.

\section{Reproducibility}

Differences between the values obtained for a given variable in a person on several occasions are due partly to analytical error and partly to biological variation. Repeated analysis of a plasma specimen with a mean creatinine concentration of about $125 \mu \mathrm{mol} / 1$ (near the upper limit of normal) gives a range of values with a standard deviation of $8 \mu \mathrm{mol} / \mathrm{l}$ and a coefficient of variation of $7 \%$ (Table 3 ). The total within-person variability is not much greater than the analytical. There is no significant diurnal variation of $\mathbf{P}$ in health (Sratland, Winkel and Bokelund, 1973a), no difference between fasting values and those after a meal, and only a small increase after even very strenuous exercise (Statland et al., 1973b).

If analytical error is the same for urine as it is for plasma and one assumes no error in the urine collection, then the analytical coefficient of variation
TABLE 3. Standard data for plasma creatinine (P) ( $\mu \mathrm{mol} / \mathrm{l})$ and creatinine clearance $(\mathrm{Ccr})(\mathrm{ml} / \mathrm{min})$ in men aged $20-30$ years

\begin{tabular}{lrrrrrr}
\hline & \multicolumn{2}{c}{$P(\mu \mathrm{mol} / \mathrm{l})$} & & \multicolumn{2}{c}{ Ccr $(\mathrm{ml} / \mathrm{min})$} \\
\cline { 2 - 3 } \cline { 6 - 7 } Mean & \multicolumn{2}{c}{88} & & \multicolumn{2}{c}{120} \\
\cline { 2 - 3 } \cline { 5 - 6 } Variation & s.d. & CV (\%) & & s.d. & CV (\%) \\
\hline Total & 13 & 15 & & 25 & 20 \\
Within-person & 9 & 10 & & 18 & 15 \\
$\quad$ Analytical & 6 & 7 & & 11 & 9 \\
Biological & 6 & 7 & & 14 & 12 \\
Between-person & 10 & 11 & & 17 & 14 \\
\hline
\end{tabular}

of Ccr is at least $10 \%$ and the standard deviation at $100 \mathrm{ml} / \mathrm{min}$ is $10 \mathrm{ml} / \mathrm{min}(95 \%$ confidence limits 80-129 $\mathrm{ml} / \mathrm{min}$ ) - see Appendix I. However, it is very difficult, if not impossible, to get complete urine collections over accurately timed periods in general medical or surgical wards or in out-patients. Any formal attempt to measure this error would involve repeated urine collection and would inevitably alter the attitude of the staff and patient to the collection 
and give estimates of error smaller than those happening in everyday practice. The reported coefficients of variation for the urinary excretion of creatinine vary between 6 and $18 \%$ (Doolan et al., 1962; Paterson, 1967; Cramer, Cramer and Selander, 1967). The coefficient of variation of $\mathrm{Ccr}$ within a person, including analytical and biological variation, is at least $15 \%$ (Table 3 ). The $95 \%$ confidence limits at an obtained value of $100 \mathrm{ml} / \mathrm{min}$ are then $70-130$ $\mathrm{ml} / \mathrm{min}$.

\section{Prediction of creatinine clearance}

Several authors have suggested that the errors and difficulties in the measurement of $\mathrm{Ccr}$ can be avoided by predicting it from plasma creatinine. The relation between $\mathrm{P}$ and $\mathrm{Ccr}$ (Fig. 1) has been made linear either by plotting the logarithms of the two variables (Effersoe, 1956) or by plotting Ccr against the reciprocal of the plasma creatinine (Edwards and Whyte, 1959; Doolan et al., 1962; Enger and Blegen, 1964; Jelliffe, 1971). This approach takes no account of the variation in the urinary excretion of creatinine between persons at a given age and weight. Kampmann et al. (1971) and Cockroft and Gault (1976) have given functions for the prediction of $\mathrm{Ccr}$ from age and weight. The error of these predictions was $21-27 \%$, which is not less than the standard deviation of the range of $\mathrm{Ccr}$ at a given age (Table 3 ) and, surprisingly, it is not much less for the predictions based on age and weight than for that based on $\mathbf{P}$ alone. However, the predictions, although inaccurate, are reproducible in the sense that the only source of variation in a series of measurements is the variation in the $\mathbf{P}$. This argument is discussed in more detail by Bröchner-Mortensen and Rödbro (1976).

\section{The detection of 'abnormal' glomerular function}

Three arguments form the basis for the widely held view that $\mathrm{Ccr}$ is preferable to $\mathbf{P}$ as a measure of glomerular function.

(a) The obvious objection to $P$ is that it is in no sense a measure of glomerular filtration rate whereas $\mathrm{Ccr}$ is closely related to GFR and changes in step with and in the same direction as the GFR.

(b) A much-used argument against $\mathbf{P}$ as an assessment of glomerular function (Ccr) has been that it is 'insensitive' since it only becomes abnormal when glomerular function is grossly reduced.

(c) Another argument is that a wide range of values of $\mathrm{Ccr}$ can be found at any particular observed value of $P$ (Fig. 1) and there will be patients who have a 'low' Ccr but a 'normal' $P$.

However, the authors suggest that in these arguments little consideration has been given to whether a statement made about a group can be applied to the individual, and, in general, it has not always been fully appreciated that what is true about a physiological variable such as GFR (often measured under ideal experimental conditions) may not be true of the imprecise estimate of that variable made in clinical practice (Ccr). An underlying factor has probably been the belief that any estimate of GFR is 'better' than the $P$ as a guide to glomerular function. In addition there has been a failure to take account of the reduction of glomerular function with age. The three objections to $P$ as a measure of glomerular function can be challenged, even for persons of a particular age and sex.

The first objection, namely that $\mathbf{P}$ is not a measure of Ccr does not carry much weight since all that matters in the present context is whether a measure 'works' in clinical practice, not whether it has an appropriate physiological basis. Indeed this same argument was used to justify the adoption of $\mathrm{Ccr}$ rather than inulin clearance (Haugen and Blegen, 1935).

The second objection is that the $P$ is insensitive and of limited use in practice because it does not become abnormal until the glomerular function falls to $25 \%$ of normal or less. This conclusion is based on reports such as those of Tobias et al. (1962) and Doolan et al. (1962) which demonstrate relationships between $P$ and Ccr similar to that shown in Fig. 1. The data have been interpreted in terms of the value of $\mathrm{Ccr}$ at which the average $\mathbf{P}$ reaches the upper limit of normal for $\mathbf{P}$. But the wide range of $\mathrm{Ccr}$ associated with a $\mathbf{P}$ at the upper limit of normal was due in part to the inclusion of older persons who have lower Ccr without a higher P (Figs 2 and 3). For persons of a given age, e.g. young men, the $P$ is more sensitive to reduction in $\mathrm{Ccr}$, in that the average $P$ reaches the upper limit of normal at a Ccr which is $75 \%$ of normal. However, amongst these young men there will be individuals whose usual value for $\mathbf{P}$ is at the upper limit of normal and whose $\mathbf{P}$ will become abnormal as soon as the Ccr falls. Other individuals whose usual $P$ is at the bottom of the normal range will not have a $\mathbf{P}$ above the normal range until the Ccr has fallen to $50 \%$ of normal. This lack of sensitivity in the detection of abnormality is present in any situation where one is comparing a patient's value with a reference range rather than with his value when he was healthy. The limitation applies to the Ccr itself since, if one takes the range in Table 2, an individual with a value at the top of the range to begin with would not have an abnormally low $\mathrm{Ccr}$ until he had lost $60 \%$ of his original glomerular function.

It is the third objection that Ccr may be low when the $\mathbf{P}$ is normal which casts most doubt on the usefulness of the P. The work of Doolan et al. (1962) and Tobias et al. (1962) did much to consolidate this view. Both are excellent studies but the conclusions 
can be challenged. Tobias et al. base their conclusions on mean values of three separate measurements of Ccr which will greatly decrease the error in the Ccr and increase its 'usefulness'. They emphasize that there were patients with a low Ccr but a $P$ within the normal range. But the converse will also happen, that is, there will also be patients with a raised $\mathbf{P}$ whose Ccr has fallen, but is still within the normal range. Indeed, among the 102 patients of Doolan et al. (1962) who had P and Ccr measured, both measures were normal in forty-five, both were abnormal in forty-two, Ccr alone was abnormal in three, but $\mathbf{P}$ alone was abnormal in twelve. Tobias et al. (1962) also emphasize that some patients have a low Ccr with a normal $P$ and they give details of five such patients. But it is clear from their Fig. 1 that there were patients with a raised $\mathbf{P}$ and a normal $\mathrm{Ccr}$. A disturbing aspect of the paper of Tobias and his colleagues (1962) is that they excluded clearances where the 'urinary output . . . was suspiciously low'.

In order to assess the relative sensitivity of $\mathbf{P}$ and $\mathrm{Ccr}$ in the detection of abnormal glomerular function the authors have taken the situation of a group of young men with the reference values of $P$ and $\mathrm{Ccr}$ given in Table 3 and calculated the distribution of $P$ and $\mathrm{Ccr}$ as Ccr progressively decreased. The plasma creatinine at each $\mathrm{Ccr}$ was calculated assuming a constant U.V (details of the calculation are given in Appendix II). Figure 5 shows the calculated frequency of persons with abnormal values for $\mathbf{P}$ and Ccr at each new average value of Ccr. The $P$ is clearly a more sensitive detector of abnormality of glomerular function. The individuals identified as abnormal differed for the two measures depending on where the initial value lay within the reference range. However, as Fig. 5 shows, only a small number of patients had an abnormal Ccr without an abnormal $P$. The calculations have been repeated on the basis of triplicate measurements of $\mathbf{P}$ and $\mathrm{Ccr}$. The $\mathbf{P}$ was still more sensitive than the Ccr (Fig. 5).

The diagnostic usefulness of the Ccr is further limited if patients of all ages are included since, unlike $\mathbf{P}$, the $\mathrm{Ccr}$ changes with age and the reference ranges according to age have not been adequately defined. Alternative measures to $\mathrm{Ccr}$, such as the clearance of ${ }^{51} \mathrm{Cr}$-EDTA are more reproducible (Bröchner-Mortersen and Rödbro, 1976) but still have the disadvantage that efficient use requires age specific reference ranges. The present advantage of these alternatives over $\mathbf{P}$, if any, will depend on their reproducibility in routine practice and the age distribution of the population which is being investigated. Indeed, if the reference range for young persons is applied to a hospital population most of the abnormal values for $\mathrm{Ccr}$ will be due to the fall in Ccr with age, and not due to renal disease.

The authors suggest, therefore, that none of the

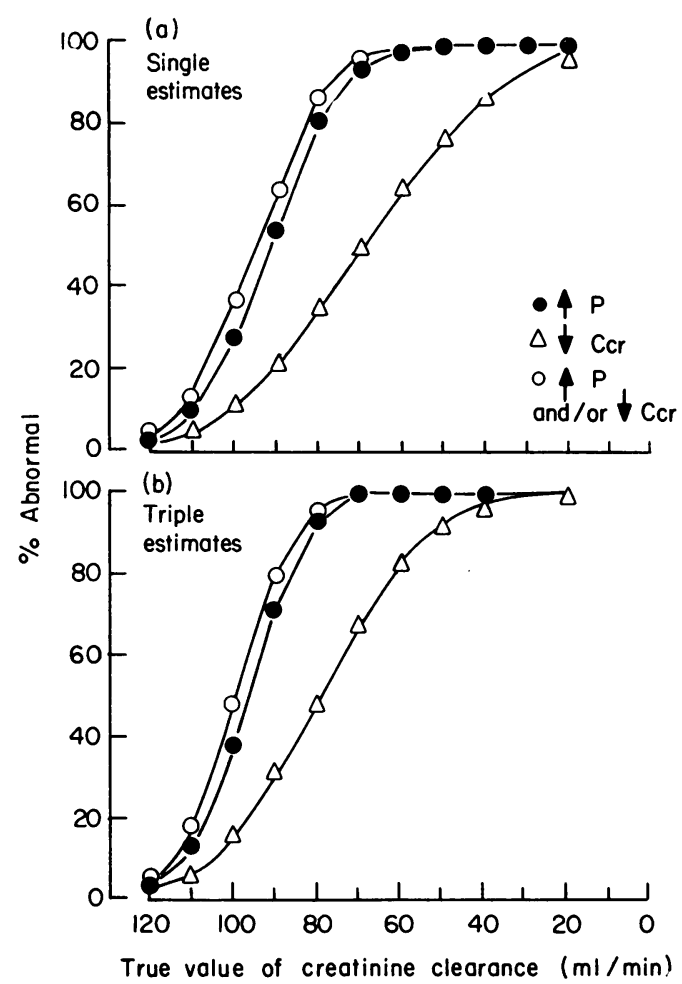

Fig. 5. The calculated frequency $(\%)$ of abnormal values for $\mathbf{P}$ and Ccr (alone or together) in a group of young men with decreased Ccr. The frequencies have been calculated for (a) single estimates, (b) the mean of triple estimates of the variables. Details of the calculation are given in Appendix II(a).

three arguments for $\mathrm{Ccr}$ and against $\mathbf{P}$ as the detector of abnormal glomerular function is valid and that, for several reasons, $P$ is to be preferred.

The detection of abnormality is very much simpler with $\mathbf{P}$ than with Ccr. Plasma creatinine is more reproducible, it is easier to replicate samples to get an estimate of the set, or reference value in an individual, there are adequate published data for $\mathbf{P}$, laboratories can readily obtain samples from appropriate groups of men and women to determine their own reference values, and there is a negligible change of the $\mathbf{P}$ with age. Interpretation is simple since a normal $\mathbf{P}$ can be taken as evidence that a patient's glomerular function is adequate for the demand on it, whether or not it has changed as a result of disease. A $\mathbf{P}$ above the reference range indicates that glomerular function is reduced.

\section{The detection of change in glomerular function}

The sensitivity of a test in detecting a change depends on how much the values for the test can vary when there is no real change in the set value 


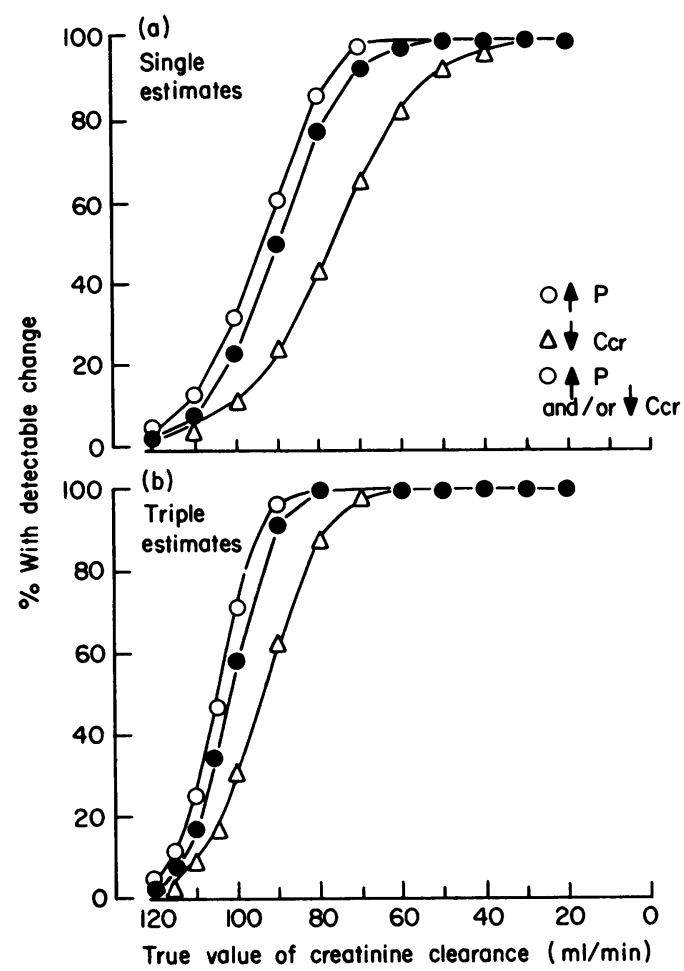

FIG. 6. The calculated frequency $(\%)$ of detectable change in $P$ and Ccr (alone or together) as the Ccr progressively decreased in a group of young men. The frequencies have been calculated for (a) single estimates and (b) the mean of triple estimates of the variables. Details of the calculations are given in Appendix II(b).

for the individual; this variation can be analytical and biological. For $\mathbf{P}$ at values near the upper limit of the normal range $(125 \mu \mathrm{mol} / \mathrm{l})$, the analytical variability is such that one would need to find a difference between successive values greater than about $27 \mu \mathrm{mol} / 1(0.3 \mathrm{mg} / 100 \mathrm{ml})$, or $22 \%$, to be reasonably confident that a patient's set value had really changed (see Table 1 , Appendix I).

The measurement of Ccr combines two measurements of creatinine concentration, $P$ and $U$. If errors in urine collection are ignored, the imprecision of Ccr must be $41 \%$ greater than that of $P$ in terms of analytical factors alone and one would need to find a change greater than $22 \mathrm{ml} / \mathrm{min}$ at $100 \mathrm{ml} / \mathrm{min}$ to be confident that there had been a real change.

If one takes the estimates of reproducibility shown in Table 3 then the change in Ccr would have to be $33 \%$ before one would be confident that a real change has happened (Appendix II). In order to compare measurements of $\mathbf{P}$ and $\mathrm{Ccr}$ as detectors of change of Ccr the authors have considered the values of $P$ and Ccr which might be observed in a man whose set values in health were $1.0 \mathrm{mg} / 100 \mathrm{ml}(88 \mathrm{~mol} / \mathrm{l})$ and
$120 \mathrm{ml} / \mathrm{min}$ respectively and whose set value for $\mathrm{Ccr}$ progressively decreased. The values for $P$ have been calculated from the values for Ccr, assuming that $\mathrm{U} . \mathrm{V}$ is constant (details of the calculation are given in Appendix II). Figure 6 shows the frequency of significantly raised $\mathbf{P}$ and lowered $\mathrm{Ccr}$ at each value of Ccr. The change is detected more frequently with $P$ than with $\mathrm{Ccr}$ and this is the case even when the calculations are based on triplicate measurements of $\mathbf{P}$ and $\mathrm{Ccr}$ (Fig. 6). $\mathbf{P}$ is therefore more sensitive than Ccr in the detection of change in glomerular function.

However, creatinine clearance does have the advantage that it changes in the direction of and in proportion to the changes in GFR, whereas $P$ does not (Fig. 1). These advantages can be given to $P$ by calculating its reciprocal. The reciprocal of $\mathbf{P}$ ( $\mu \mathrm{mol} / \mathrm{l})$ multiplied by $10^{4}$ has the same magnitude as $\mathrm{Ccr}(\mathrm{ml} / \mathrm{min})$. Creatinine excretion is said to decrease in severe renal failure which would apparently make $\mathbf{P}$ (or its reciprocal) unreliable assessments of the progress of the renal failure. However, this decrease is small and the reciprocal of $\mathbf{P}$ continues to decrease linearly with time in individual patients even as renal failure becomes terminal (Mitch et al., 1976; Rutherford et al., 1977).

\section{Conclusion}

The authors suggest that, in order to assess a patient's glomerular function, one does not really need to know the GFR but rather whether the GFR is appropriate for the patient's age and physique, and thus whether he is able to maintain his internal environment within acceptable limits. This information is provided by the plasma creatinine. It is true that a reduction in glomerular function can happen without the $P$ becoming abnormal (i.e. outside the normal range) but this happens even more frequently for $\mathrm{Ccr}$, and deterioration in glomerular function is detected less frequently with $\mathrm{Ccr}$ than with $P$. The reciprocal of plasma creatinine has the further advantages that it changes in the same direction as, and in proportion to, the changes in glomerular function. The authors believe the routine measurement of Ccr should be abandoned, and efforts should be directed to narrowing the reference ranges for $P$ by taking account of body size and composition. These efforts are already being made in paediatrics (Counahan et al., 1976).

\section{Appendix I}

The CV for a single measurement rises for two independent measurements, $a$ and $b$,to $\sqrt{\mathrm{CV}^{2}{ }_{a}+\mathrm{CV}^{2}{ }_{\mathrm{h}}}$, an increase of $41 \%$ if $\mathrm{CV}_{\mathrm{a}}=\mathrm{CV}_{\mathrm{b}}$. Since the $\mathrm{CV}$ of $\mathrm{P}$ is about $7 \%$, the $\mathrm{CV}$ of the urine to plasma creatinine concentration ratio (U/P), a part of the calculation of Ccr, is about $\sqrt{7^{2}+7^{2}=10 \%}$. 
A patient's measurement can be considered to have changed significantly (at $P<0.05)$ if the difference between successive values exceeds 2.7 s.d., where s.d. is the standard deviation of repeated measurements separated by the same interval as the patient's values (Campbell and Owen, 1967; Payne, 1972).

If one includes all sources of error (Table 3) the $\mathrm{CV}$ of $\mathrm{Ccr}$ is $15 \%$ and the difference between two values would have to exceed $33 \mathrm{ml} / \mathrm{min}$ before it could be regarded as a significant change (at $P$ 0.05).

\section{Appendix II}

Comparison of $\mathbf{P}$ and $\mathrm{Ccr}$ in assessment of glomerular function.

\section{(a) Detection of abnormal glomerular function}

The calculations were based on a group of young men with the mean and range of $P$ and $C c r$ shown in Table 3. The distribution of each variable was assumed to be Gaussian. The mean $\mathrm{Ccr}$ was then reduced by $10 \mathrm{ml} / \mathrm{min}$ (s.d. unchanged), the U.V was kept constant and the mean and s.d. of the 'new' $\mathbf{P}$ were calculated. Abnormal values of $\mathrm{Ccr}$ were taken as values below the $95 \%$ limit of the original distribution and abnormal values for $\mathbf{P}$ were taken as values above the $95 \%$ limit of the original distribution. In order to calculate the frequency $(\%)$ of abnormal values of $\mathbf{P}$ and $\mathrm{Ccr}$ when the $\mathrm{Ccr}$ was reduced by $10 \mathrm{ml} / \mathrm{min}$, the position of the limit of normality within the new distribution of values was defined in terms of deviation from the mean in standard deviation units. The percentage of the population outside this limit of the distribution was obtained from tables. These calculations were repeated for progressive reduction in Ccr.

In order to enable the comparison on the basis of the mean of triplicate measurements it was assumed that the standard deviation of the within-person variability would be reduced by a factor of $\sqrt{3}$. The s.d. of the distribution in the group of young men was calculated on this basis.

\section{(b) Detection of change}

This calculation was based on a man with the set values for $\mathbf{P}$ and $\mathbf{C r}$ which were the means of the reference population of young men (Table 3). Even if there was no real change in the set value the observed values of $\mathbf{P}$ and Ccr could vary from time to time within distributions with the within-person standard deviations shown in Table 3. The true Ccr of this man was reduced by $10 \mathrm{ml} / \mathrm{min}$. The new within-person variation was calculated assuming that the analytical coefficient of variation was unchanged. The new set value for $P$ was calculated on the assumption that the U.V was unchanged. The within-person variability around this set value was calculated on the basis that the within-person coefficient of variation had not changed. The distribution of differences between single values from the original and new distributions was calculated.

The percentage of this distribution of differences with values greater than twice the standard deviation of the difference was calculated. These calculations were repeated for progressive decreases of $\mathrm{Ccr}$, and on the basis of the mean of triplicate measurements of $\mathbf{P}$ and $\mathrm{Ccr}$.

\section{References}

Bröchner-Mortensen, J. \& RöDbro, P. (1976) Selection of routine method for determination of glomerular filtration rate in adult patients. Scandinavian Journal of Clinical and Laboratory Investigation, 36, 35.

Campbell, D.G. \& Owen, J.A. (1967) Clinical laboratory error in perspective. Clinical Biochemistry, $1,3$.

Cockroft, D.W. \& Gault, M.H. (1976) Prediction of creatinine clearance from serum creatinine. Nephron, 16, 31.

Cook, J.G.H. (1975) Factors influencing the assay of creatinine. Annals of Clinical Biochemistry, 12, 219.

Counahan, R., Chantler, C., Ghazali, S., Kirkwood, B., Rose, R. \& BARRATT, T.M. (1976) Estimation of glomerular filtration rate from plasma creatinine concentration in children. Archives of Disease in Childhood, 51, 875.

Cramer, K., Cramer, H. \& Selander, S. (1967) A comparative analysis between variation in 24-hour urinary creatinine output and 24-hour urinary volume. Clinica chimica acta, 15, 331.

Donckerwolcke, R.A.M.G., Sander, P.C., van StekelenBURG, G.J., STOOP, J.W. \& TIDDENS, H.A.W.M. (1970) Serum creatinine values in healthy children. Acta paediatrica scandinavica, 59, 339.

Doolan, P.D., AlPen, E.L. \& TheIl, G.B. (1962) A clinical appraisal of the plasma concentration and endogenous clearance of creatinine. American Journal of Medicine, 32, 65.

EDWARDS, K.D.G. \& WHYTE, H.M. (1959) Creatinine excretion and body composition. Clinical Science, 18, 361.

EFfersoe, P. (1956) Relationship between endogenous 24 hour creatinine clearance and serum creatinine concentration in patients with chronic renal disease. Acta medica scandinavica, 156, 429.

ENGER, E. \& BlEgEN, E.M. (1964) The relationship between endogenous creatinine clearance and serum creatinine in renal failure. Scandinavian Journal of Clinical and Laboratory Investigation, 16, 273.

Haugen, H.N. \& Blegen, E.M. (1935) Plasma 'creatinine' concentration and creatinine clearance in clinical work. Annals of Internal Medicine, 43, 731 .

JeLlifFE, R.W. (1971) Estimation of creatinine clearance when urine cannot be collected. Lancet, i, 975.

Kampmann, J.P., Sierbaek-Nielsen, K., Kristensen, M. \& HANSEN, J.M. (1971) Aldersbetingede variationer i urinkreatinin og endogen kreatininclearance. Ugeskrift for La:ger, 133, 2369.

KASSIRER, J.P. (1971) Clinical evaluation of kidney function glomerular function. New England Journal of Medicine, 285, 385.

KERR, D.N.S. \& DAVISON, J.M. (1975) The assessment of renal function. British Journal of Hospital Medicine, October, 360.

de lauture, H., Caces, E., Durost, O., Tournier, M., Dolle, Y., Weill, J., Boulard, P. \& Rossier, J. (1973) In: Reference Values in Human Chemistry, p. 141. S. Krager, Basel.

McIntosh, J.F., Moller, E. \& van Slyke, D.D. (1928) The influence of body size on urea output. Journal of Clinical Investigation, 6, 467. 
MATERSON, B.J. (1971) Measurement of glomerular filtration rate. CRC Critical Reviews in Clinical Laboratory Sciences, January, 1.

Mitch, W.E., Buffington, G.A., Walser, M. \& LemanN $J_{R}$, J. (1976) A simple method of estimating progression of chronic renal failure. Lancet, ii, 1326.

Paterson, N. (1967) Relative constancy of 24-hour urine volume and 24 -hour creatinine output. Clinica chimica acta, 18, 57.

PAYNe, R.B. (1972) The application of standard deviation calculated from successive duplicates to quality control data. Clinica chimica acta, 42, 263.

Payne, R.B. \& Morgan, D.B. (1977) Sodium, water and acid-base balance: teaching transient and steady states. Medical Education, 11, 133.

Rowe, J.W., ANDres, R., Tobin, J.D., NorRis, A.H. \& SHock, N.W. (1976) The effect of age on creatinine clearance in men: a cross sectional and longitudinal study. Journal of Gerontology, 31, 155.

Rutherford, W.E., Blandin, J., Miller, J.P., Greenwalt, A.S. \& VARRA, J.D. (1977) Chronic prognosis of renal disease: rate of change of serum creatinine concentrations. Kidney International, 11, 62.
Schwartz, G.J., Haycock, G.B., Chir, B. \& Spitzer, A. (1976) Plasma creatinine and urea concentration in children: normal values for age and sex. Journal of Pediatrics, 88, 828.

Statland, B.E., Winkel, P. \& Bokelund, H. (1973a) Factors contributing to intra-individual variation of serum constituents: 1 . Within-day variation of serum constituents in healthy subjects. Clinical Chemistry, 19, 1374.

Statland, B.E., Winkel, P. \& Bokelund, H. (1973b) Factors contributing to intra-individual variation of srum constituents: 2 . Effects of exercise and diet on variation of serum constituents in healthy subjects. Clinical Chemistry, $19,1380$.

Tobias, G.J., Mclaughlin, R.F. \& Hopper, J. (1962) Endogenous creatinine clearance. New England Journal of Medicine, 266, 317.

Vuhlback, B., Eriksson, A. \& Forsius, H. (1964) Plasma creatinine in different sex and age groups of a healthy isolated island population. Acta medica scandinavica, 412 (suppl.), 83.

Wilding, P., Rollason, J.G. \& Robinson, D. (1972) Patterns of change for various biochemical constituents detected in well population screening. Clinica chimica acta, 41, 375. 